\title{
Middle ear microbiome differences in indigenous Filipinos with chronic otitis media due to a duplication in the A2ML1 gene
}

Regie Lyn P. Santos-Cortez ${ }^{1, *^{*}}$, Diane S. Hutchinson ${ }^{2}$, Nadim J. Ajami², Ma. Rina T. Reyes-Quintos ${ }^{3,4}$, Ma. Leah C. Tantoco ${ }^{3}$, Patrick John Labra ${ }^{4}$, Sheryl Mae Lagrana ${ }^{3}$, Melquiadesa Pedro ${ }^{3}$, Erasmo Gonzalo d. V. Llanes ${ }^{3,4}$, Teresa Luisa Gloria-Cruz ${ }^{3,4}$, Abner L. Chan ${ }^{3,4}$, Eva Maria Cutiongco-de la Paz, ${ }^{5,6}$, John W. Belmont ${ }^{7,10}$, Tasnee Chonmaitree ${ }^{8}$, Generoso T. Abes ${ }^{3,4}$, Joseph F. Petrosino ${ }^{2}$, Suzanne M. Leal ${ }^{1}$ and Charlotte M. Chiong ${ }^{3,4}$

\begin{abstract}
Background: Previously rare A2ML1 variants were identified to confer otitis media susceptibility in an indigenous Filipino community and in otitis-prone US children. The goal of this study is to describe differences in the middle ear microbiome between carriers and non-carriers of an A2ML1 duplication variant that increases risk for chronic otitis media among indigenous Filipinos with poor health care access.
\end{abstract}

Methods: Ear swabs were obtained from 16 indigenous Filipino individuals with chronic otitis media, of whom 11 carry the A2ML1 duplication variant. Ear swabs were submitted for 165 rRNA gene sequencing.

Results: Genotype-based differences in microbial richness, structure, and composition were identified, but were not statistically significant. Taxonomic analysis revealed that the relative abundance of the phyla Fusobacteria and Bacteroidetes, and genus Fusobacterium were nominally increased in carriers compared to non-carriers, but were non-significant after correction for multiple testing. We also detected rare bacteria including Oligella that was reported only once in the middle ear.

Conclusions: These findings suggest that A2ML1-related otitis media susceptibility may be mediated by changes in the middle ear microbiome. Knowledge of middle ear microbial profiles according to genetic background can be potentially useful for therapeutic and prophylactic interventions for otitis media and can guide public health interventions towards decreasing otitis media prevalence within the indigenous Filipino community.

Keywords: $A 2 M L 1$, Indigenous population, Microbiome, Middle ear, Oligella, Otitis media, Philippines Abbreviations: OTU, Operational taxonomic units; PCR, Polymerase chain reaction

\footnotetext{
* Correspondence: regie.santos-cortez@ucdenver.edu

${ }^{1}$ Department of Molecular and Human Genetics, Center for Statistical

Genetics, Baylor College of Medicine, Houston, TX 77030, USA

${ }^{9}$ Current affiliation: Department of Otolaryngology, University of Colorado

School of Medicine, Aurora, CO 80045, USA

Full list of author information is available at the end of the article
} 


\section{Multilingual abstracts}

Please see Additional file 1 for translations of the abstract into the six official working languages of the United Nations.

\section{Background}

Otitis media or middle ear infection is an important public health problem worldwide. In developed countries otitis media remains the top reason for health care visits and antibiotic use among children [1], while incidence and prevalence of otitis media are particularly high in sub-Saharan Africa, Asia and in marginalized communities such as indigenous populations, where the burden of complications due to chronic otitis media (e.g., hearing loss) is mostly felt [2]. Due to high prevalence of chronic suppurative otitis media and its complications in developing countries, it was recently proposed for chronic otitis media to be classified as a neglected tropical disease [3]. While multiple risk factors are associated with otitis media, there is strong evidence that a genetic basis for otitis media susceptibility exists $[4,5]$.

Recently we identified rare variants in the A2ML1 gene, which encodes alpha-2-macroglobulin-like 1 protein, as a cause of otitis media susceptibility [6]. A rare A2ML1 duplication variant c.2478_2485dupGGCTAAAT (p.(Ser829Trpfs"9)) confers susceptibility to otitis media in three European- or Hispanic-American children in Texas, USA and an indigenous Filipino population. This variant co-segregated with different forms of otitis media in a six-generation pedigree within the indigenous Filipino community, which is highly intermarried due to socio-economic segregation and cultural discrimination and has a relatively homogeneous environmental background i.e., poor health care access and hygiene, lack of pneumococcal vaccination, crowded households and swimming in dirty seawater. In this population, known risk factors for otitis media including quantitative age, gender, nutrition and tobacco exposure were not associated with otitis media status [7]. Additionally the three US children who also carry the A2ML1 duplication variant had early-onset recurrent otitis media that required tympanostomy tube insertion within the first six months of age. The duplication variant occurred within a short haplotype that was common among the indigenous Filipinos and the US children, suggesting a founder variant that is estimated to be 1800 years old and that may have occurred within the Filipino and US populations through Spanish colonization [6].

Genetic susceptibility to otitis media has been established using family and association studies, however the mechanisms by which genetic variants influence hostbacterial interactions in the middle ear has not been elucidated. Here we show suggestive evidence that carriage of the $A 2 M L 1$ duplication variant might influence the middle ear microbial composition of individuals with chronic otitis media, which may explain in part the pathogenic mechanism by which the $A 2 M L 1$ duplication variant confers otitis media susceptibility. This study is novel for several reasons: (A) Most microbiome studies for (non-chronic) otitis media used nasopharyngeal and adenoid samples rather than middle ear fluid or swabs, and differences in microbial profiles according to sample source within the head and neck is known $[8,9]$. (B) Previously middle ear microbiome studies were performed for chronic otitis media using a single sample and on 11 indigenous Australian children with effusive otitis media $[10,11]$. (C) Comparison of microbiome findings according to human host genotype has only been done for a few diseases (e.g., cystic fibrosis, inflammatory bowel disease), but not otitis media [12, 13]. This study also further supports the concept that in the presence of human or host mutation, the complexity of disease patterns can be partly attributed to changes in the microbiome.

\section{Methods}

The study was approved by the: Baylor College of Medicine (BCM) Institutional Review Board and Affiliated Hospitals; the National Commission on Indigenous Peoples, Philippines; and the University of the Philippines Manila Research Ethics Board. Informed consent was obtained from adult participants and parents or guardians of pediatric patients. Participating individuals from an indigenous Filipino community were examined by otoscopy. Previous analyses of multiple risk factors for otitis media established a homogeneous environmental background for this community [7]. Despite high prevalence of otitis media, this community has poor access to health care including vaccinations and antibiotic treatments and has no surgical facilities within the island. In many cases, otitis media either spontaneously resolves with age or results in chronic eardrum perforation with recurrent discharge. Chronically perforated eardrums result in long-term exposure of the middle ear mucosa to the outer ear flora and the environment, e.g., during bathing or swimming in the sea.

From each individual, human genomic DNA from saliva was obtained using Oragene DNA saliva kits (DNA Genotek, Ontario, Canada). DNA samples were Sanger-sequenced for the A2ML1 duplication variant [6]. From indigenous Filipinos with perforated eardrum(s) due to chronic otitis media, outer ear swabs were collected prior to middle ear swabs, using sterile short polyester-tipped Pur-Wrap swabs (Puritan Medical, Guilford, ME, USA). The outer ear swabs were rubbed gently against outer ear canal skin, then additional swabs were rubbed against middle ear mucosa and/or edges of eardrum perforations and soaked on discharge when 
present. Each swab specimen was placed directly in the collection tube from the PowerSoil DNA isolation kit (MO BIO Laboratories, Carlsbad, CA, USA), the swab stem cut with sterile scissors, and the collection tube closed, sealed and labeled. All samples were stored in a $-20{ }^{\circ} \mathrm{C}$ freezer until shipped to $\mathrm{BCM}$ on dry ice.

All collected swabs were submitted to the BCM Alkek Center for Metagenomics and Microbiome Research for $16 \mathrm{~S}$ rRNA gene profiling. Bacterial genomic DNA was isolated from ear swabs using the PowerSoil DNA isolation kit. 16S rRNA gene sequencing methods were adapted from the NIH-Human Microbiome Project. Briefly, the 16S rRNA gene V4 region was amplified by PCR and sequenced on an Illumina MiSeq $(2 \times 250 \mathrm{bp})$. The primers (515F and 806R) used for amplification contain adapters for MiSeq sequencing and single-index barcodes allowing PCR products to be pooled and sequenced directly $[14,15]$. The read pairs were demultiplexed based on the unique molecular barcodes and filtered at an expected error rate of 0.05 . Reads were truncated at first instance of Q5 and then size selected between 252-254 bp. Reads were merged using USEARCH v7.0.1001 [16] using an 8 bp seed sequence, at least $50 \mathrm{bp}$ of overlap, and zero mismatches across the overlap. The 16S rRNA gene sequencing analysis pipeline incorporates phylogenetic and alignment-based approaches to maximize data resolution. 16S rRNA gene sequences were assigned into Operational Taxonomic Units (OTUs) at a similarity cutoff value of $97 \%$ using the UPARSE algorithm [16]. OTUs were mapped at $97 \%$ sequence identity to an optimized version of the SILVA 123 database, containing only the V4 region to determine taxonomy. Abundances were recovered by mapping demultiplexed reads to the UPARSE OTUs. Sequences were normalized to 10000 sequences per sample.

The microbial sequence data was analyzed for $\alpha$ diversity, which measures richness, evenness, or diversity of individual samples, and $\beta$-diversity, which measures dissimilarity between samples based on phylogenetic distance and taxonomic composition [17]. Because multiple swabs were available per individual, outer ear swabs and middle ear swabs were analyzed separately. For bilateral samples, swabs from the right ear were included in analyses. The PERMANOVA test was applied to $\beta$-diversity metrics to ascertain differences in community composition between wildtype individuals and $A 2 M L 1$ variant carriers. Differential abundance of taxa was detected by the Mann-Whitney test with false discovery rate (FDR, Benjamini-Hochberg) correction for multiple comparisons $(P<0.05$ significance threshold). 16S rRNA gene compositional analysis provided a summary of the composition and structure of the bacterial component of the microbiome. Due to our previous findings that aside from the $A 2 M L 1$ duplication no other risk factors were associated with otitis media status within the indigenous Filipino community [7], no other variables were used in the analyses.

\section{Results}

Middle and outer ear swabs were obtained from 16 indigenous Filipino individuals (Table 1), 11 of whom were carriers of the $A 2 M L 1$ variant. Median age was 9.5 years, and females comprised $62.5 \%$ of study subjects. Half of the individuals with middle ear samples were bilaterally affected. Clinical history and otoscopic findings from follow-up over 2-10 years indicated chronic otitis media for all individuals examined, with the last examination revealing well-defined edges of eardrum perforations ranging from $20 \%$ to near-total perforations. Description of discharge when present varied widely, being copious or scanty with moist middle ear mucosa, purulent or mucoid, foul- or non-foul-smelling. When visualized, the middle ear mucosa was usually thickened. In three ears, granulation tissue was observed. Also two individuals had chronic otitis media in one ear, but acute or effusive otitis media in the other ear. According to local health office records, none of the 16 indigenous Filipinos with chronic otitis media had vaccinations for common pathogens for acute otitis media, i.e., Streptococcus pneumoniae or Haemophilus influenzae, or recent antibiotic use at time of examination.

Middle and outer ear swabs were available from a total of 21 ears with chronic otitis media from 16 indigenous Filipinos (Table 1; Additional file 2: Figure S1). The median DNA concentration per ear swab was $3.0 \mathrm{\eta g} / \mu \mathrm{l}$ (maximum $92.5 \mathrm{\eta g} / \mu \mathrm{l}$ ), indicating that these samples contain high bacterial biomass. For five individuals with bilateral samples, middle ear microbial profiles were not different between ears (Additional file 3: Figure S2), thus for these five individuals only the right ear was included in comparisons by genotype. When comparing middle ears of wildtype versus $A 2 M L 1$-positive individuals, observed OTUs were not different (Additional file 2: Figure $\mathrm{S} 1 \mathrm{~A})$. The mean Shannon diversity index was higher in middle ears of carriers, however the difference was not statistically significant (Additional file 2: Figure S1B). Similar plots were also non-significant for the outer ears (Additional file 4: Figure S3). The $\beta$-diversity analyses for middle ear swabs were not statistically significant between carriers and non-carriers (Additional file 2: Figures S1C and S1D). Lack of significant results for these metrics may be largely due to the small sample size.

At phylum level, relative taxa abundance profiles for the middle ear differed by genotype, with more Fusobacteria and Bacteroidetes in carriers of the $A 2 M L 1$ variant than in non-carriers (Table 2, Fig. 1a). At genus level, Fusobacterium was also greater in carriers (Table 3, Fig. 1b). The higher relative abundance for these taxa 
Table 1 Clinical data and A2ML1 duplication variant genotypes of indigenous Filipinos with ear swabs

\begin{tabular}{llllll}
\hline ID & Age (yrs) & Sex & GT & Side & Otoscopic findings \\
\hline 2 & 10 & F & wt & L & $20 \%$ perforation and copious yellowish foul-smelling discharge; reddish dull TM \\
5 & 10 & M & het & B & $60-80 \%$ perforation with purulent foul-smelling discharge \\
7 & 4 & M & het & R & $30 \%$ perforation with purulent thick non-foul-smelling discharge \\
8 & 24 & F & wt & L & $20 \%$ perforation, dry \\
20 & 6 & F & het & R & $20 \%$ perforation, scanty non-foul-smelling discharge \\
33 & 9 & M & wt & B & near-total perforation with thick purulent non-foul-smelling discharge \\
41 & 10 & F & het & R & $70 \%$ perforation with moist middle ear mucosa \\
45 & 19 & F & het & B & B: $20 \%$ perforation with yellowish non-foul-smelling discharge: L: granulation tissue \\
53 & 14 & F & het & B & $30-40 \%$ perforation with discharge \\
62 & 6 & M & hom & R & $20 \%$ perforation with non-foul-smelling discharge \\
63 & 13 & F & hom & B & $40-90 \%$ perforation with moist middle ear mucosa \\
67 & 6 & F & het & L & $20 \%$ perforation, dry \\
71 & 12 & F & wt & L & $50 \%$ perforation with moist middle ear mucosa \\
76 & 7 & M & wt & B b & L: $30 \%$ perforation with discharge; R: intact but retracted TM \\
89 & 4 & F & het & B b & R: foul-smelling discharge; L: dull, bulging erythematous TM \\
92 & 7 & M & het & B & filled with granulation tissue and foul-smelling discharge \\
\hline
\end{tabular}

GT genotype, $F$ female, $M$ male, wt wildtype, het heterozygous, hom homozygous, $L$ left, $R$ right, $B$ bilateral, $T M$ tympanic membrane

aD 45 has chronic otitis media bilaterally but middle ear sample is available only for the right ear

${ }^{\mathrm{b}}$ Perforation only in one ear, for which a middle ear sample was obtained

was nominally significant in carriers $(P \leq 0.05)$, but nonsignificant after correction for multiple testing which is not unexpected given the small sample size. Based on identified genera the composition of the middle ear microbiome in the A2ML1 variant carriers differed from non-carriers due to higher relative abundance in Fusobacterium, Porphyromonas, Peptostreptococcus, Parvimonas and Bacteroides (Table 3, Fig. 1b), while median frequencies for Alloiococcus, Staphylococcus, Proteus and Haemophilus were greater in wildtype middle ears (Table 3). These genera show concordance with identified phyla, e.g., higher relative abundance of the phyla Fusobacteria and Bacteroidetes in middle ears of A2ML1-positive individuals due to greater median frequencies for genera Fusobacterium and Porphyromonas/Bacteroides, respectively (Fig. 1; Table 3). On the other hand, non-significant increases in relative abundance were detected for the phyla Proteobacteria (i.e., due to Proteus, Haemophilus, Alcaligenaceae or Pseudomonas) and Actinobacteria (i.e.,
Brevibacterium, Actinomyces or Corynebacterium) in middle ears of non-carriers (Tables 2 and 3).

For outer ears the unweighted UniFrac principal coordinate analysis was significant by genotype although weighted UniFrac analysis was non-significant (Additional file 4: Figure S3). The bacterial taxa identified in the outer ears were similar to middle ear bacteria (Tables 4, 5, Additional file 2: Figure S1 and Additional file 4: Figure S3). Overall these findings suggest similarity in middle and outer ear bacteria due to cross-contamination across chronically perforated eardrums. This is further supported by the lack of detection of Bacteroidetes and/or Fusobacteria in previous microbiome studies of the external auditory canals of healthy individuals $[18,19]$.

\section{Discussion}

These microbiome results provide some insight into the effect of the A2ML1 duplication variant on otitis media susceptibility [6]. Even though $\alpha$ - and $\beta$-diversity

Table 2 Bacterial phyla in middle ears of indigenous Filipinos with chronic otitis media

\begin{tabular}{lccccc}
\hline Phylum & Overall median \% & A2ML1+ median \% & Wildtype median \% & Mann- Whitney $p$ & FDR-adjusted $p^{\text {a }}$ \\
\hline Fusobacteria & 2.72 & 9.15 & 0.04 & 0.04 & 0.24 \\
Bacteroidetes & 13.51 & 28.03 & 0.16 & 0.05 & 0.24 \\
Proteobacteria & 36.31 & 29.59 & 52.75 & 0.15 & 0.44 \\
Actinobacteria & 12.40 & 6.98 & 33.28 & 0.51 & 0.74 \\
Firmicutes & 3.96 & 3.94 & 4.30 & 0.83 & 0.83 \\
\hline
\end{tabular}

${ }^{\mathrm{a}}$ False discovery rate (FDR) 


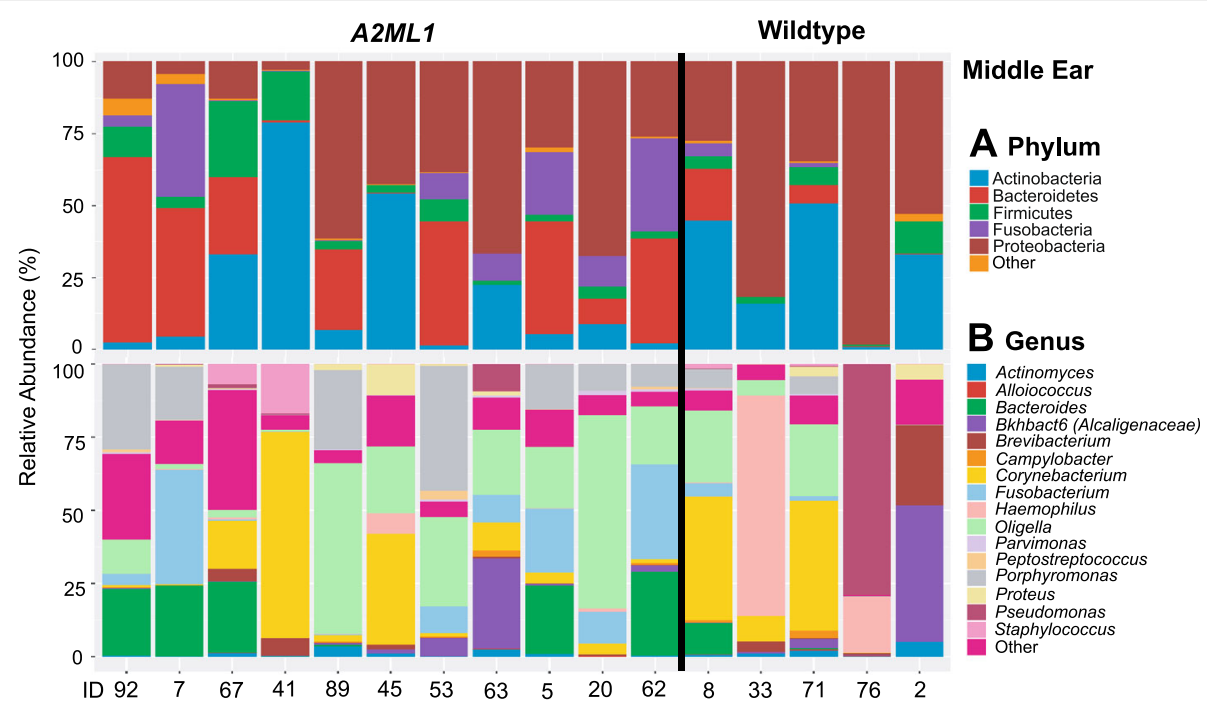

Fig. 1 Relative abundance of middle ear bacterial taxa according to A2ML1 genotype. a By phylum; b by genus

measurements were not significantly different in $A 2 M L 1$ variant carriers versus non-carriers, there is suggestive evidence that carriage of the $A 2 M L 1$ variant and the expected loss-of-function of A2ML1 protein might favor survival and growth of specific bacterial taxa. At genus level, Alloiococcus was among the top three bacteria identified from the middle ear, although at very low frequency (Table 3). Alloiococcus otitidis was initially discovered in middle ear fluid [20]. On the other hand, Fusobacterium and Porphyromonas had greater relative abundance in $A 2 M L 1$ variant carriers compared to non- carriers at $\sim 9 \%$ median frequency in the middle ear (Table 3). The species Fusobacterium nucleatum and Porphyromonas gingivalis are less commonly isolated in chronic otitis media [21] but are known to proliferate in oropharyngeal infections [21] or progressive gingivitis and periodontitis which are mucosal diseases in the oral cavity [22]. During infection, these two bacteria along with host inflammatory cells release proteases that degrade mucosal matrix and basement membrane, and of these two bacteria, $P$. gingivalis can produce higher proteolytic activity and elicit a more virulent host

Table 3 Middle ear bacterial genera with $\geq 0.1 \%$ difference in relative abundance by genotype

\begin{tabular}{|c|c|c|c|c|c|}
\hline Phylum & Genus & Overall median \% & $A 2 M L 1+$ median $\%$ & Wildtype median \% & Mann- Whitney $p^{a}$ \\
\hline Fusobacteria & Fusobacterium & 2.72 & 9.15 & 0.04 & 0.05 \\
\hline Firmicutes & Alloiococcus & 0 & 0 & 0.24 & 0.06 \\
\hline Bacteroidetes & Porphyromonas & 6.22 & 9.00 & 0.06 & 0.07 \\
\hline Firmicutes & Staphylococcus & 0.03 & 0.01 & 0.11 & 0.11 \\
\hline Firmicutes & Peptostreptococcus & 0.05 & 0.19 & 0.01 & 0.14 \\
\hline Firmicutes & Parvimonas & 0.19 & 0.21 & 0.01 & 0.21 \\
\hline Proteobacteria & Proteus & 0.10 & 0 & 0.16 & 0.24 \\
\hline Bacteroidetes & Bacteroides & 0.36 & 0.69 & 0.08 & 0.30 \\
\hline Proteobacteria & Haemophilus & 0.17 & 0.07 & 0.41 & 0.31 \\
\hline Proteobacteria & Oligella & 20.48 & 21.07 & 5.33 & 0.38 \\
\hline Actinobacteria & Brevibacterium & 0.41 & 0.22 & 0.66 & 0.40 \\
\hline Actinobacteria & Actinomyces & 0.73 & 0.50 & 1.11 & 0.44 \\
\hline Proteobacteria & Bkhbact6 (Alcaligenaceae) & 0.51 & 0.44 & 0.65 & 0.61 \\
\hline Proteobacteria & Campylobacter & 0.19 & 0.29 & 0 & 0.73 \\
\hline Proteobacteria & Pseudomonas & 0.06 & 0.03 & 0.15 & 0.77 \\
\hline Actinobacteria & Corynebacterium & 3.78 & 3.74 & 8.62 & 1 \\
\hline
\end{tabular}

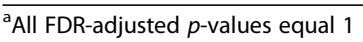


Table 4 Bacterial phyla in outer ears of indigenous Filipinos with chronic otitis media

\begin{tabular}{lcclcc}
\hline Phylum & Overall median $\%$ & A2ML1+ median $\%$ & Wildtype median \% & Mann- Whitney $p$ & FDR-adjusted $p^{\text {a }}$ \\
\hline Bacteroidetes & 2.97 & 32.25 & 0.05 & 0.009 & 0.07 \\
Fusobacteria & 1.56 & 3.11 & 0 & 0.04 & 0.16 \\
Proteobacteria & 34.89 & 34.56 & 75.20 & 0.27 & 0.36 \\
Firmicutes & 3.49 & 3.63 & 0.59 & 0.44 & 0.50 \\
Actinobacteria & 15.07 & 11.97 & 24.39 & 0.91 & 0.91 \\
\hline
\end{tabular}

response [23]. The protein structure of A2ML1 is highly homologous to the protease inhibitor alpha-2macroglobulin (A2M), and we previously hypothesized that A2ML1 and A2M might have overlapping protective roles within the middle ear by preventing undue mucosal damage from either bacterial or inflammatory proteases [6]. Interestingly, there is evidence that human A2M efficiently inhibits some cysteine proteases produced by $P$. gingivalis, but another homologous macroglobulin is required to trap all proteinase forms and control proteolytic activity due to $P$. gingivalis [24]. A defect in macroglobulin function in the middle ear mucosa may favor infection with Porphyromonas or Fusobacterium through unregulated bacterial protease activity.

While Porphyromonas and Fusobacterium were previously isolated in cultures of ear discharge from chronic otitis media [21, 25], these bacterial groups that are more abundant in the $A 2 M L 1$-variant carriers are quite different from major bacterial isolates from chronic middle ear discharge from the general Filipino population, e.g., Staphylococcus, Pseudomonas, Proteus [26]. Our microbiome results are also different from a previous microbiome study which largely detected Pseudomonas from middle ear fluid of a US child with chronic otitis media [10]. In another microbiome study of middle ear fluid from indigenous Australian children with effusive otitis media, the top species detected were Alloiococcus otitidis, Haemophilus influenzae and Streptococcus sp. [11] which are more frequently detected in non-chronic forms of otitis media i.e., acute or effusive otitis media [27]. Note however that Haemophilus influenzae has also been identified in chronic otitis media and is known to form biofilm in the middle ear [28]. Although the uniqueness of the bacterial profiles in indigenous Filipinos vs. other populations may be attributed to differences in sampling and detection techniques, type of otitis media and community setting, the differences in diversity and abundant taxa by genotype as described here were detected while comparing indigenous Filipino individuals only. All the indigenous Filipinos studied were diagnosed

Table 5 Outer ear bacterial genera with $\geq 0.1 \%$ difference in relative abundance by genotype

\begin{tabular}{|c|c|c|c|c|c|}
\hline Genus & Overall median \% & A2ML1+ median \% & Wildtype median \% & Mann- Whitney $p^{a}$ & $\overline{\text { FDR-adjusted } p}$ \\
\hline Porphyromonas & 1.69 & 7.62 & 0.02 & 0.01 & 0.30 \\
\hline Fusobacterium & 1.56 & 3.11 & 0 & 0.04 & 0.39 \\
\hline Haemophilus & 0.42 & 0.23 & 9.61 & 0.04 & 0.39 \\
\hline Parvimonas & 0.11 & 0.18 & 0 & 0.06 & 0.45 \\
\hline Bacteroides & 0.25 & 1.26 & 0.03 & 0.09 & 0.53 \\
\hline Campylobacter & 0.08 & 0.18 & 0 & 0.11 & 0.53 \\
\hline Peptostreptococcus & 0.06 & 0.19 & 0.01 & 0.15 & 0.54 \\
\hline Proteus & 0.19 & 0.03 & 0.50 & 0.28 & 0.63 \\
\hline Peptoniphilus ${ }^{a}$ & 0.17 & 0.17 & 0.07 & 0.32 & 0.65 \\
\hline Corynebacterium & 7.83 & 3.81 & 12.00 & 0.38 & 0.71 \\
\hline Pseudoclavibacter ${ }^{b}$ & 1.56 & 0.66 & 2.13 & 0.38 & 0.71 \\
\hline Oligella & 22.88 & 27.23 & 6.85 & 0.44 & 0.75 \\
\hline Bkhbact6 (Alcaligenaceae) & 0.62 & 0.83 & 0.21 & 0.58 & 0.75 \\
\hline Alloiococcus & 0.04 & 0 & 0.11 & 0.59 & 0.75 \\
\hline Dietzia $^{b}$ & 0.15 & 0.01 & 0.24 & 0.60 & 0.75 \\
\hline Brevibacterium & 0.95 & 1.17 & 0.72 & 0.66 & 0.78 \\
\hline
\end{tabular}

Phylum information: ${ }^{\mathrm{a}}$ Firmicutes; ${ }^{\mathrm{b}}$ Actinobacteria 
with chronic otitis media, had clinical history of several years of inadequately treated chronic ear discharge, and were from the same homogeneous community, providing supportive evidence that the microbial profile changes are more likely due to genotype. Moreover the finding of known oral cavity pathogens as the predominant middle ear bacteria in $A 2 M L 1$ variant carriers begs further study of a potential link between oral health and otitis media status in these individuals.

For the indigenous Filipino community, microbiome technologies have high utility given the lack of access to laboratory facilities including microbial culture. The identification of bacteria that were rarely or never reported in middle ears also testifies to the utility of $16 \mathrm{~S}$ rRNA gene profiling in detecting bacteria that may be missed in culture studies. Oligella was reported only once in ear discharge [29] while the OTU BkhBact6 (Alcaligenaceae), which was classified to genus GKS98 freshwater group of the class Betaproteobacteria, has only been seen in environmental samples [30]. Bkhbact6 (Alcaligenaceae) had higher relative abundance in the middle ears of wildtype individuals (Table 3). Oligella was highly abundant in both middle and outer ears and was occasionally the predominant genus in the middle ear swabs (Table 3, Fig. 1b). In addition, the relative abundance of Oligella was greater than that of Corynebacterium, which has been isolated from middle and outer ears but considered debatable in terms of pathogenic potential [31]. Although Oligella sp. is known as a commensal in the genitourinary tract, it was previously isolated from deep tissue infection and might also be considered an opportunistic pathogen [29]. Currently the paucity of literature on Oligella and Bkhbact6 (Alcaligenaceae) precludes conclusion if these bacteria are middle ear pathogens, commensals or contaminants.

\section{Conclusions}

Overall our findings suggest that the $A 2 M L 1$ duplication variant may induce changes in the middle ear microbiome. It should be stressed that the study was designed to be exploratory rather than associative, therefore these findings are descriptive in nature and require confirmation using a larger number of subjects. Nevertheless, this study highlights the importance of host genotypebacterial interactions in understanding the pathophysiology of common infectious diseases such as otitis media. Specifically, within the indigenous Filipino community, knowledge of middle ear microbial profiles according to genetic background can be potentially useful for future therapeutic and prophylactic interventions, such as antibiotic use and vaccination that includes coverage against Haemophilus which was detected by 16S rRNA sequencing.

\section{Additional files}

Additional file 1: Multilingual abstracts in the six official working languages of the United Nations. (PDF $763 \mathrm{~kb}$ )

Additional file 2: Figure S1. Middle ear microbial profiles of indigenous Filipinos with chronic otitis media. All panels compare carriers with non-carriers of the A2ML1 duplication variant. Panel description: (A) a-diversity by observed OTUs; (B) a-diversity by the Shannon diversity index; (C) $\beta$-diversity from unweighted UniFrac principal coordinate analysis; (D) $\beta$-diversity from weighted UniFrac principal coordinate analysis. (PDF $1019 \mathrm{~kb}$ )

Additional file 3: Figure S2. Comparison of right and left ears from five individuals with bilateral ear samples. Plots comparing the most abundant phyla and genera in the outer and middle ears show very similar profiles between right and left ears. Blue bars represent taxa that are more abundant in the right ear, green bars for the left ear. Only phylum Firmicutes $(P=0.02)$ and genus Alcaligenaceae which belongs to phylum Proteobacteria $(P=0.02)$ were significantly different between right and left outer ear swabs from these five individuals, however $p$ values were non-significant for all comparisons after correction for multiple testing. For the five individuals with bilateral samples, only right ear swabs were included in microbial analyses by genotype. (PDF 750 kb)

Additional file 4: Figure S3. Outer ear microbial profiles by genotype. Panel description as in Additional file 2: Figure S1. (PDF 783 kb)

\section{Acknowledgments}

We thank the members of the indigenous Filipino community for study participation. We are grateful to: Xin Wang and Izoduwa Abbe for Sanger sequencing of DNA samples; Tulin Ayvaz from the CMMR for her work in sample processing; and Ginger Metcalf, Donna Muzny, and Richard Gibbs from the BCM Human Genome Sequencing Center for their support in sequencing.

\section{Funding}

This work was supported by: the National Organization for Hearing Research Foundation, the Hearing Health Foundation, and Action On Hearing Loss (to R.L.P.S.C.); the University of the Philippines Manila - National Institutes of Health (to G.T.A.); the Albert and Margaret Alkek Foundation (to J.F.P.); and the United States National Institutes of Health - National Institute on Deafness and Other Communication Disorders grants K18 DC013564 (to T.C.), R01 DC011651 and R01 DC003594 (to S.M.L.) and R01 DC015004 (to R.L.P.S.C.).

\section{Availability of data and materials}

Microbiome data from this study are being submitted to the National Center for Biotechnology Information (NCBI) Sequence Read Archive (SRA).

\section{Authors' contributions}

RLPSC, conception of study design, data collection, analysis and interpretation, manuscript writing, final approval, accountable for all aspects of the work; DSH, data analysis and interpretation, manuscript writing, final approval, accountable for all aspects of the work; NJA, data analysis and interpretation, manuscript writing, final approval, accountable for all aspects of the work; MRTRQ, conception of study design, data collection, manuscript writing, final approval, accountable for all aspects of the work; MLCT, data collection, manuscript writing, final approval, accountable for all aspects of the work; PJL, data collection, manuscript writing, final approval, accountable for all aspects of the work; ShML, data collection, manuscript writing, final approval, accountable for all aspects of the work; MP, data collection, manuscript writing, final approval, accountable for all aspects of the work; EGdVL, data collection, manuscript writing, final approval, accountable for all aspects of the work; TLGC, data collection, manuscript writing, final approval, accountable for all aspects of the work; ALC, data collection, manuscript writing, final approval, accountable for all aspects of the work; EMCdIP, data collection, manuscript writing, final approval, accountable for all aspects of the work; JWB, conception of study design, manuscript writing, final approval, accountable for all aspects of the work; TC, data analysis and interpretation, manuscript writing, final approval, accountable for all aspects of the work; GTA, data collection, manuscript writing, final approval, 
accountable for all aspects of the work; JFP, data analysis and interpretation, manuscript writing, final approval, accountable for all aspects of the work; SuML, conception of study design, analysis and interpretation, manuscript writing, final approval, accountable for all aspects of the work; CMC, conception of study design, data collection, manuscript writing, final approval, accountable for all aspects of the work.

\section{Competing interests}

J.W.B. is an employee of Illumina, Inc. N.J.A. and J.F.P. own shares in Diversigen Inc., a microbiome research company. All authors declare no conflict of interest.

\section{Ethics approval and consent to participate}

The study was approved by the: BCM Institutional Review Board and Affiliated Hospitals (protocol H-27321); the National Commission on Indigenous Peoples, Philippines (ADO-CP-XI-08-06-692); and the University of the Philippines Manila Research Ethics Board (UPMREB 2007-031-01). Informed consent was obtained individually from adult participants and parents or guardians of pediatric patients.

\section{Author details}

'Department of Molecular and Human Genetics, Center for Statistical Genetics, Baylor College of Medicine, Houston, TX 77030, USA. ${ }^{2}$ Department of Molecular Virology and Microbiology, Alkek Center for Metagenomics and Microbiome Research (CMMR), Baylor College of Medicine, Houston, TX 77030, USA. ${ }^{3}$ Philippine National Ear Institute, University of the Philippines Manila - National Institutes of Health (UPM-NIH), Manila 1000, Philippines. ${ }^{4}$ Department of Otorhinolaryngology, University of the Philippines College of Medicine - Philippine General Hospital, Manila 1000, Philippines. ${ }^{5}$ Institute of Human Genetics, UPM-NIH, Manila 1000, Philippines. ${ }^{6}$ Philippine Genome Center, University of the Philippines, Diliman, Quezon City 1101, Philippines. ${ }^{7}$ Departments of Molecular and Human Genetics and Pediatrics, Baylor College of Medicine, Houston, TX 77030, USA. ${ }^{8}$ Division of Pediatric Infectious Disease and Immunology, Department of Pediatrics, University of Texas Medical Branch, Galveston, TX 77555, USA. ${ }^{9}$ Current affiliation: Department of Otolaryngology, University of Colorado School of Medicine, Aurora, CO 80045, USA. ${ }^{10}$ Current address: Illumina, Inc, San Diego, CA 92122, USA.

\section{Received: 25 May 2016 Accepted: 26 August 2016}

Published online: 01 November 2016

\section{References}

1. Vaz LE, Kleinman KP, Raebel MA, Nordin JD, Lakoma MD, Dutta-Linn MM, Finkelstein JA. Recent trends in outpatient antibiotic use in children. Pediatrics. 2014;133:375-85.

2. Monasta L, Ronfani L, Marchetti F, Montico M, Vecchi Brumatti L, Bavcar A, Grasso D, Barbiero C, Tamburlini G. Burden of disease caused by otitis media: systematic review and global estimates. PLoS One. 2012;7:e36226.

3. Li MG, Hotez PJ, Vrabec JT, Donovan DT. Is chronic suppurative otitis media a neglected tropical disease? PLoS Negl Trop Dis. 2015;9:e0003485.

4. Daly KA, Brown WM, Segade F, Bowden DW, Keats BJ, Lindgren BR, Levine SC, Rich SS. Chronic and recurrent otitis media: a genome scan for susceptibility loci. Am J Hum Genet. 2004;75:988-97.

5. Allen EK, Chen WM, Weeks DE, Chen F, Hou X, Mattos JL, Mychaleckyj JS, Segade F, Casselbrant ML, Mandel EM, Ferrell RE, Rich SS, Daly KA, Sale MM. A genome-wide association study of chronic otitis media with effusion and recurrent otitis media identifies a novel susceptibility locus on chromosome 2. J Assoc Res Otolaryngol. 2000;14:791-800.

6. Santos-Cortez RLP, Chiong CM, Reyes-Quintos MRT, Tantoco MLC, Wang X, Acharya A, Abbe I, Giese AP, Smith JD, Allen EK, Li B, la Paz EM C-de, Garcia MC, Llanes EG, Labra PJ, Gloria-Cruz TL, Chan AL, Wang GT, Daly KA, Shendure J, Bamshad MJ, Nickerson DA, Patel JA, Riazuddin S, Sale MM, University of Washington Center for Mendelian Genomics, Chonmaitree T, Ahmed ZM, Abes GT, Leal SM. Rare A2ML1 variants confer susceptibility to otitis media. Nat Genet. 2015:47:917-20.

7. Santos-Cortez RLP, Reyes-Quintos MRT, Tantoco MLC, Abbe I, Llanes EGdV, Ajami NJ, Hutchinson DS, Petrosino JF, Padilla CD, Villarta RL, Gloria-Cruz TL, Chan AL, Cutiongco-de la Paz EM, Chiong CM, Leal SM, Abes GT. Genetic and environmental determinants of otitis media in an indigenous Filipino population. Otol Head Neck Surg 2016; doi:10.1177/0194599816661703.
8. Hilty M, Qi W, Brugger SD, Frei L, Agyeman P, Frey PM, Aebi S, Mühlemann K. Nasopharyngeal microbiota in infants with acute otitis media. J Infect Dis. 2012;205:1048-55

9. Ren T, Glatt DU, Nguyen TN, Allen EK, Early SV, Sale MM, Winther B, Wu M. 165 rRNA survey revealed complex bacterial communities and evidence of bacterial interference on human adenoids. Environ Microbiol. 2013;15:535-47.

10. Liu CM, Cosetti MK, Aziz M, Buchhagen JL, Contente-Cuomo TL, Price LB, Keim PS, Lalwani AK. The otologic microbiome: a study of the bacterial microbiota in a pediatric patient with chronic serous otitis media using 16SrRNA gene-based pyrosequencing. Arch Otolaryngol Head Neck Surg. 2011;137:664-8

11. Jervis-Bardy J, Rogers GB, Morris PS, Smith-Vaughan HC, Nosworthy E, Leong LE, Smith RJ, Weyrich LS, De Haan J, Carney AS, Leach AJ, O'Leary S, Marsh $\mathrm{RL}$. The microbiome of otitis media with effusion in Indigenous Australian children. Int J Pediatr Otorhinolaryngol. 2015;79:1548-55.

12. Cox MJ, Allgaier M, Taylor B, Baek MS, Huang YJ, Daly RA, Karaoz U, Andersen GL, Brown R, Fujimura KE, Wu B, Tran D, Koff J, Kleinheinz ME, Nielson D, Brodie EL, Lynch SV. Airway microbiota and pathogen abundance in age-stratified cystic fibrosis patients. PLoS One. 2010:5:e11044

13. Spor A, Koren O, Ley R. Unravelling the effects of the environment and host genotype on the gut microbiome. Nat Rev Microbiol. 2011;9:279-90.

14. Caporaso JG, Lauber CL, Walters WA, Berg-Lyons D, Lozupone CA, Turnbaugh PJ, Fierer N, Knight R. Global patterns of $16 \mathrm{~S}$ rRNA diversity at a depth of millions of sequences per sample. Proc Natl Acad Sci USA. 2011; 108 Suppl 1:4516-22.

15. Caporaso JG, Lauber CL, Walters WA, Berg-Lyons D, Huntley J, Fierer N, Owens SM, Betley J, Fraser L, Bauer M, Gormley N, Gilbert JA, Smith G, Knight R. Ultra-high-throughput microbial community analysis on the Illumina HiSeq and MiSeq platforms. ISME J. 2012;6:1621-4.

16. Edgar RC. UPARSE: highly accurate OTU sequences from microbial amplicon reads. Nat Methods. 2013;10:996-8.

17. Lozupone C, Lladser ME, Knights D, Stombaugh J, Knight R. UniFrac: an effective distance metric for microbial community comparison. ISME J. 2011:5:169-72.

18. Costello EK, Lauber CL, Hamady M, Fierer N, Gordon Jl, Knight R. Bacterial community variation in human body habitats across space and time. Science. 2009;326:1694-7.

19. Frank DN, Spiegelman GB, Davis W, Wagner E, Lyons E, Pace NR. Cultureindependent molecular analysis of microbial constituents of the healthy human outer ear. J Clin Microbiol. 2003:41:295-303.

20. Aguirre M, Collins MD. Phylogenetic analysis of Alloiococcus otitis gen. nov., sp. nov., an organism from human middle ear fluid. Int J Syst Bacteriol. 1992:42:79-83.

21. Suzuki K, Kurono Y, Ikeda K, Watanabe A, Iwamoto A, Totsuka K, Kaku M, Iwata S, Kadota J, Hanaki H. Nationwide surveillance of 6 otorhinolaryngological infectious diseases and antimicrobial susceptibility pattern in the isolated pathogens in Japan. J Infect Chemother. 2015;21:483-91.

22. Yang NY, Zhang Q, Li JL, Yang SH, Shi Q. Progression of periodontal inflammation in adolescents is associated with increased number of Porphyromonas gingivalis, Prevotella intermedia, Tannerella forsynthesis and Fusobacterium nucleatum. Int J Paediatr Dent. 2014;24:226-33.

23. Jie Bao G, Kari K, Tervahartiala T, Sorsa T, Meurman JH. Proteolytic activities of oral bacteria on proMMP-9 and the effect of synthetic proteinase inhibitors. Open Dent J. 2008:2:96-102.

24. Grøn H, Pike R, Potempa J, Travis J, Thøgersen IB, Enghild JJ, Pizzo SV. The potential role of alpha 2-macroglobulin in the control of cysteine proteinases (gingipains) from Porphyromonas gingivalis. J Periodontal Res. 1997:32(1 Pt 1):61-8.

25. Brook I. The role of anaerobic bacteria in chronic suppurative otitis media in children: implications for medical therapy. Anaerobe. 2008;14:297-300.

26. del Rosario R, Chiong CM, Chan AL, Yap EC, Jamir JC, Abes GT. Microbial flora in chronic otitis media: value of ear aspirate culture studies. Philipp J Otolaryngol Head Neck Surg. 1990;1:58-66.

27. Holder RC, Kirse DJ, Evans AK, Peters TR, Poehling KA, Swords WE, Reid SD. One third of middle ear effusions from children undergoing tympanostomy tube placement had multiple bacterial pathogens. BMC Pediatr. 2012;12:87.

28. Van Hoecke H, De Paepe AS, Lambert E, Van Belleghem JD, Cools P, Van Simaey L, Deschaght P, Vaneechoutte M, Dhooge I. Haemophilus influenzae biofilm formation in chronic otitis media with effusion. Eur Arch Otorhinolaryngol. 2016; doi:10.1007/s00405-016-3958-9 
29. Rossau R, Kersters K, Falsen E, Jantzen E, Segers P, Union A, Nehls L, de Ley J. Oligella, a new genus including Oligella urethralis comb. nov. (formerly Moraxella urethralis) and Oligella ureolytica sp. nov. (formerly CDC Group IVe): relationship to Tay/orella equigenitalis and related taxa. Int J Syst Bacteriol. 1987;37:198-210

30. Wu L, Ge G, Zhu G, Gong S, Li S, Wan J. Diversity and composition of the bacterial community of Poyang Lake (China) as determined by $16 \mathrm{~S}$ rRNA gene sequence analysis. World J Microbiol Biotechnol. 2012;28:233-44.

31. von Graevenitz A, Funke G. Turicella otitidis and Corynebacterium auris: 20 years on. Infection. 2014;42:1-4.

Submit your next manuscript to BioMed Central and we will help you at every step:

- We accept pre-submission inquiries

- Our selector tool helps you to find the most relevant journal

- We provide round the clock customer support

- Convenient online submission

- Thorough peer review

- Inclusion in PubMed and all major indexing services

- Maximum visibility for your research

Submit your manuscript at www.biomedcentral.com/submit
Biomed Central 